\title{
A Clinico-Epidemiological Study of Leprosy in an Urban Leprosy Centre of West Bengal
}

\author{
Dr. Jayanta Kumar Barua, Dr. Deep Anurag, Dr. Goutam Barik, Dr. Prerna, \\ Dr. Loknath Ghoshal, Dr. Devesh Kumar Shukla, Dr. Gautam Banerjee
}

\begin{abstract}
A Clinico epidemiological study of 208 patients of leprosy who sought medical advice at the Leprosy OPD of School of Tropical Medicine, Kolkata, during January 2016 - April 2016 is reported. Among all the cases, $94.2 \%$ were new cases, $3.8 \%$ were defaulters and $1.9 \%$ were having a relapse after completion of treatment. Around 59\% of them had borderline tuberculoid type. An important finding was that prevalence of $B L \& L L$ cases before the age of 50 years is about $15.8 \%$ but beyond this age the prevalence rises to $47.6 \%$. Depending on slit skin smear report 146 (70.2\%) cases were multibacillary leprosy. The disease was found 2.3 times more often in males than in females and was found mainly in the age group 11-70 years. Lepra reactions were seen in 17 (8.17\%) cases, of which 10 had type 1 reaction (58.8\%) and 7 type 2 reaction (41.2\%). Another significant finding was the occurrence of lepra reactions (both type I \& II) in multibacillary type of leprosy $(n=14)$ than in paucibacillary type. Among the 146 patients with multibacillary disease 42 (28.76\%) developed deformity and most (80.95\%) would have more severe and permanent deformities.
\end{abstract}

Keywords: Leprosy, Paucibacillary, Multibacillary, Deformities, Lepra reaction.

\section{Introduction}

Leprosy is a chronic disease caused by Mycobacterium leprae. It is an infectious disease mainly affecting the peripheral nerves. It also affects the skin, muscles, eyes, bones, testes and internal organs. ${ }^{[1]}$ The National Leprosy Control Programme was launched in 1954 in India and converted to National Leprosy Elimination Programme (NLEP) in 1983 with the objective to eliminate leprosy. ${ }^{[1]}$ The South East Asian region accounts for about $66.21 \%$ of the global prevalence and $71 \%$ of all new detected cases. ${ }^{[2]}$ With the introduction of MDT(Multi Drug Treatment) since 1983, India has achieved the goal of elimination of leprosy in December $2005 .{ }^{[3]} \mathrm{A}$ total of 0.92 lakh cases are on record as on 1st April 2013, giving a Prevalence rate (PR) of 0.73 per 10,000 population ${ }^{[4]}$ The aim of present study was to find out the clinical presentations of leprosy patients in an urban leprosy centre (ULC) in West Bengal from January 2016-April 2016, and to interpret this data with respect to different epidemiological variables like age, sex, type of disease, deformity, reaction etc.

\section{Material \& Methods}

For this study, case records of patients attending Leprosy OPD of School of Tropical Medicine, Kolkata between January 2016 to April 2016 were analysed. Their age, sex, mode of presentation, history of any household contacts, presence or absence of any deformities, history of treatment and the occurrence of any subsequent lepra reaction was noted.

Patient with clinical signs of leprosy who had not received multi-drug therapy previously, was called a "New" case. Any patient with leprosy, who had received MDT previously but had not completed the therapy, was called a "Defaulter".

When a patient developed new signs and symptoms of the disease even after receiving an adequate course of MDT, was called a "Relapse" case.

Deformities when present were graded based on WHO grading system. If neither anaesthesia, nor any visible deformity was present, it was called Grade 0 . Grade 1 deformity signified the presence of anaesthesia alone. Grade 2 signified presence of visible deformities like claw hand, foot drop etc.

The cases were classified clinically according to Ridley and Jopling classification. A further classification into multi-bacillary and pauci-bacillary cases was done after review of the slit-skin smear report. Depending upon whether they had received treatment previously or not they were classified as a new case or as a defaulter. Cases of lepra reaction are subdivided into type I and type II cases, depending upon the clinical manifestations.

Sudden appearance of erythematous and raised plaques in pre-existing lesions or appearance of new lesions, development of neuritis while under chemotherapy was categorised as type I (reversal) reaction. Development of crops of painful erythematous papules along with fever and other systemic manifestations was categorised as Erythema nodosum leprosum or type II lepra reaction. 


\section{Results}

Out of two hundred eight leprosy cases attending Leprosy OPD, 63 occurred in females and the rest in males (M:F $=2.30: 1)$. Among these 208 patients $32(15 \%)$ were clinically diagnosed as pure tuberculoid (TT) type, 123(59\%) as borderline tuberculoid (BT) type, 39(19\%) as borderline lepromatous (BL) type and 14(7\%) as pure lepromatous (LL) type. No case of mid borderline or purely neuritic type of leprosy was found. [Table 1] Depending on slit skin smear report $146(70.2 \%)$ cases were multibacillary leprosy and $62(29.8 \%)$ cases were paucibacillary leprosy.

Among the 208 patients 196 were new cases, 8 people were defaulters and 4 were relapses. All defaulter and relapse cases had a history of receiving chemotherapy. Among the four relapse cases, two presented with pure lepromatous type and two with BL type of disease. Three of the defaulter cases presented with LL type, 3 BT type and 2 with BL type of leprosy. All the patients who had relapse had multibacillary type of disease, as did the defaulters.

Among the 123 borderline tuberculoid patients, in 39(32\%) cases slit skin smear report showed paucibacillary type of leprosy. The rest (68\%) were multibacillary type of leprosy. All the Borderline lepromatous cases or pure lepromatous cases were multibacillary type on slit skin smear. However three (9\%) cases, which were clinically diagnosed as pure tuberculoid type of leprosy, showed multibacillary type on slit skin smear and received chemotherapy for a period of twelve months.[Table 2] One among them was in paediatric age group.

The distribution of various types of leprosy in different age groups is given in [Table 3].The most important finding was that prevalence of BL \& LL cases before the age of 50 years is about $15.8 \%$ but beyond this age the prevalence rises to $47.6 \%$.

Among the 208 leprosy cases 8 cases occurred in the paediatric age group, all the cases were either pure tuberculoid (37\%) or borderline tuberculoid (63\%) type, on slit skin smear examination $6(75 \%)$ were found to be paucibacillary type and the rest $2(25 \%)$ multibacillary type.[Table 4]

All TT cases were associated with involvement of a single body region, face and neck being the most common site of involvement $(n=13)$. On the other hand LL cases involved more than one body site, with face $(n=14)$ being involved in all cases and trunk involved in the majority $(\mathrm{n}=10)$. Leg is the most commonly involved site in BL cases $(n=20)$ followed by trunk $(n=15)$. Upper $(n=64)$ \& lower extremities $(n=54)$ are most commonly involved in BT cases and involvement of face \& neck $(\mathrm{n}=14)$ is less common.

At the initial presentation 48 patients presented with various kinds of deformities. Twelve $(5.7 \%)$ patients presented with anaesthesia of the involved region. Among them $11(91.7 \%)$ had BT type and $1(8.3 \%)$ had BL type of disease. The single BL case and 7 BT cases were found to be multibacillary type $(66.66 \%)$ on slit-skin smear.

Thirty six $(17.3 \%)$ other patients presented with more severe deformities; which ranges from trophic ulcer, claw hand, foot drop to lagophthalmos. The occurrence of these deformities and their association with various types of leprosy are given in [Table 5].

Presence of anaesthesia and other more severe forms of deformity are classified as grade $1 \&$ grade 2 respectively.

During the course of chemotherapy 17 patients developed either type I $(n=10)$ or type II $(n=7)$ lepra reaction. Type I reaction predominantly occurred in BT cases $(n=9), 6$ patients out of these nine had multibacillary type of leprosy. Seven patients developed type II lepra reaction. Four out of these seven had LL type and three BL type of leprosy. All had multibacillary type of disease microscopically. Altogether $4 \mathrm{BL}$ patients developed lepra reaction.[Table 6]

Another significant finding was the occurrence of lepra reactions (both type I \& II) in multibacillary type of leprosy $(n=14)$ than in paucibacillary type.

\section{Discussion}

Between the months of January 2016 and April 2016, we received 208 patients in School of Tropical Medicine, Kolkata, with a male female ratio of approximately 2.3:1, clearly showing a male preponderance. Around $70.2 \%$ of people suffered from multibacillary leprosy. Arora et al reported 63-69\% cases of multibacillary leprosy. ${ }^{[5]}$

Among all the cases $94.2 \%$ were new cases $3.8 \%$ were defaulters and $1.9 \%$ was having a relapse after completion of treatment. All of the defaulter and relapse cases had a history of receiving MDT. Although the clinical presentation in such cases varied from pure lepromatous through borderline tuberculoid type, all had multibacillary type of leprosy, emphasizing the significance of both the presence of an effective immune response and the continuation of MDT for the recommended duration, for cure of the disease.

The distribution of leprosy cases in different age group follows a normal distribution curve, with few cases occurring at the extremes of age and greater number of cases occurring between 20-50 years of age. 
However the occurrence of BL and LL cases do not follow this distribution pattern. Before the age of 50 years BL and LL cases represent $15.8 \%$ of total leprosy cases; the prevalence rises to $47.6 \%$ beyond this age.

When we compare the clinical diagnosis with microbiological one, it becomes apparent that a significant number of cases which were clinically diagnosed as BT patients have multibacillary leprosy $(68 \%)$. All these patients required 12 months of treatment with MDT for cure, emphasizing the role of a microscopical diagnosis in BT leprosy. On the other hand all the cases that were clinically diagnosed as BL or LL cases clinically had multibacillary type of leprosy. One intriguing finding was the presence of a small number of pure tuberculoid cases $(n=3)$ who were multibacillary type on microscopy. One explanation could be that such cases were diagnosed early and would eventually develop into borderline tuberculoid cases unless treated.

About $5.7 \%$ and $17.3 \%$ patients presented with Grade 1 (anaesthesia) or Grade 2 deformities respectively on initial presentation; $8.65 \%$ presented with trophic ulcer, $2.8 \%$ presented with foot drop and $4.8 \%$ with claw hand and $0.96 \%$ with lagophthalmos. $39(81.25 \%)$ cases where deformities were present were clinically diagnosed as BT patients and $9(18.75 \%)$ cases as BL patients. TT or LL patients did not present with any deformities. It did not show any male preponderance. Grade 1 and Grade 2 deformities were associated with multibacillary disease in $66.7 \%$ cases and $94.4 \%$ cases respectively.

Among the 62 cases of paucibacillary leprosy only $6(9.67 \%)$ cases developed any kind of deformity and when they did, majority $(66.66 \%)$ would have only anaesthesia. On the other hand among the 146 patients with multibacillary disease $42(28.76 \%)$ developed deformity and most $(80.95 \%)$ would have more severe and permanent deformities.

This indicates that patients with multibacillary leprosy are more prone to develop permanent deformities early in the disease.

Type I lepra reaction occurred twice as frequently in females $(7.93 \%)$ than males $(3.44 \%)(\mathrm{M}: \mathrm{F}=1: 1)$. Whereas type II lepra reaction was more common in males (4.14\%) than females (1.59\%); the male: female ratio being $6: 1$ in this case.

Significantly $14(82.4 \%)$ patients out of those 17 who developed any kind of lepra reaction had multibacillary disease, making it again a possible indicator to the development of lepra reaction. ${ }^{[6]}$ However this association didn't prove to be statistically significant, as we thought it would be. The only association of any statistical significance $(\mathrm{P}=0.0005)$ lies between the diagnosis of LL disease with the occurrence of type II lepra reaction in these patients.[table 7]

\section{Conclusion}

This study on leprosy patients attending Leprosy OPD of School of Tropical Medicine, Kolkata, corroborated several findings of other studies conducted in similar socio-economic group, which includes the distribution of disease, prevalence of deformities and their association with different types of leprosy and the association of lepra reaction with certain gender etc. This study also throws new light in showing that these deformities and lepra reaction are occurring with an increased frequency in patients with multibacillary type of disease.

\section{References}

[1]. Park JE,Park K. Text Book Of Preventive and Social Medicine,22 ${ }^{\text {nd }}$ Edition, M/S. Banarsidas Bhanot, Jabalpur;2013:287-302.

[2]. World Health Organization(2013),Weekly Epidemiological Record, No.35,30th Aug.,2013:365-380. Available from: http://www.who.int/wer. (Last Accessed on 20.01.2014)

[3]. National Institute of Epidemiology \& Indian Council of Medical Research. Report on national Workshop on surveillance of leprosy, 20 sept 2005.Chennai,India:National Institute of Epidemiology.p37.

[4]. Govt. of India (2013), National Leprosy Eradication Programme, Progress report for the year 2012-13. Dept. of Deputy Director General Leprosy,DGHS, New Delhi. .Available from:http://www.nlep.nic.in (Last accessed on17.01.2014).

[5]. Arora M, Matoch K, Natrajan M, Kamal R, Yadav VS. Changing profile of disease in leprosy patients diagnosed in a tertiary care centre during years 1995-2000.Indian J Lepr 2008; 80:257-65.

[6]. Kumar B,Dogra S, Kaur I.Epidemiological characteristics of leprosy reaction:15 years experience from North India. Int J Lepr Other Mycobact Dis 2004; 72:125-33.

Table 1

\begin{tabular}{|l|r|}
\hline Distribution of leprosy types \\
\hline TT & 32 \\
\hline BT & 123 \\
\hline BB & 0 \\
\hline BL & 39 \\
\hline LL & 14 \\
\hline PNL & 0 \\
\hline Total & 208 \\
\hline
\end{tabular}


Table 2

\begin{tabular}{|l|l|l|l|l|l|l|l|}
\hline Distribution of leprosy types \\
\hline & TT & BT & BB & BL & LL & PNL & Total \\
\hline Multibacillary & 3 & 90 & 0 & 39 & 14 & 0 & 146 \\
\hline Paucibacillary & 29 & 33 & 0 & 0 & 0 & 0 & 62 \\
\hline Total & 32 & 123 & 0 & 39 & 14 & 0 & 208 \\
\hline
\end{tabular}

Table 3

\begin{tabular}{l} 
Distribution of leprosy types according to age \\
\hline \\
\hline
\end{tabular}

Table 4

\begin{tabular}{|l|r|}
\hline \multicolumn{2}{|c|}{ Distribution of cases in paediatric age group } \\
\hline TT & 3 \\
\hline BT & 5 \\
\hline BB & 0 \\
\hline BL & 0 \\
\hline LL & 0 \\
\hline PNL & 0 \\
\hline Total & 8 \\
\hline
\end{tabular}

Table 5

\begin{tabular}{|l|l|l|l|l|l|l|l|l|}
\hline Deformities associated with types of leprosy \\
\hline & TT & BT & BB & BL(MB) & LL(MB) & PNL & Total \\
\hline Grade I & 0 & 11 & 0 & 1 & 0 & 0 & 12 \\
\hline Anaesthesia & 0 & 15 & 0 & 3 & 0 & 0 & 18 \\
\hline Grade II & 0 & 0 & 4 & 0 & 2 & 0 & 0 & 6 \\
\hline Tropic ulcer & 0 & 8 & 0 & 2 & 0 & 0 & 10 \\
\hline Foot drop & 0 & 1 & 0 & 1 & 0 & 0 & 2 \\
\hline Claw hand & & & & & \\
\hline Lagopthalmos & 0 &
\end{tabular}

Table 6

\begin{tabular}{|c|c|c|c|c|c|c|c|}
\hline \multicolumn{8}{|c|}{ Association of lepra reaction with type of disease } \\
\hline & TT(PB) & TT(MB) & $\mathrm{BT}(\mathrm{PB})$ & BT(MB) & BL(MB) & LL(MB) & Total \\
\hline Type I & 0 & 0 & 3 & 6 & 1 & 0 & 10 \\
\hline Type II & 0 & 0 & 0 & 0 & 3 & 4 & 7 \\
\hline
\end{tabular}

Table 7

\begin{tabular}{|l|l|l|l|l|}
\hline Chi -sqare analysis & \multicolumn{3}{|l|}{} \\
\cline { 1 - 4 } & BT(PB+MB) & Other & SUM & \\
\cline { 1 - 3 } Type I & 9 & 1 & 10 & \multirow{3}{*}{ P value $=0.0921$} \\
\hline No reaction & 114 & 77 & 191 & \\
\hline SUM & 123 & 78 & 201 & \\
\hline
\end{tabular}

\begin{tabular}{|l|l|l|l|l|}
\hline & LL(MB) & Other & SUM & \multirow{2}{*}{ P value $=0.0005$} \\
\hline Type II & 4 & 3 & 7 & \\
\cline { 1 - 4 } No reaction & 10 & 181 & 191 & \\
\hline SUM & 14 & 184 & 198 & \\
\hline
\end{tabular}

